\title{
Erratum: Superconductivity in the repulsive Hubbard model: An asymptotically exact weak-coupling solution [Phys. Rev. B 81, 224505 (2010)]
}

\author{
S. Raghu, S. A. Kivelson, and D. J. Scalapino \\ (Received 5 December 2016; published 19 December 2016)
}

DOI: 10.1103/PhysRevB.94.219903

According to the labels in Figs. 6, 7, and 10-12 of the published paper, what is being plotted is $V_{\text {eff }} t / U^{2}$ versus electron concentration, but in fact what is shown is the quantity $\left(V_{\text {eff }} / \rho\right) t / U^{2}$. Here $V_{\text {eff }}$ is the effective pairing potential, $U$ is the Hubbard interaction, $t$ is the nearest-neighbor hopping matrix element, and $\rho$ is the density of states at the Fermi level. None of the conclusions of the paper are affected by this error.

We are grateful to W. Cho, Y. Deng, and R. Thomale for bringing this to our attention. 